\title{
Efficient face recognition based on weighted matrix distance metrics and 2DPCA algorithm
}

\author{
CHAHRAZED ROUABHIA and HICHAM TEBBIKH
}

\begin{abstract}
In this paper, a new similarity measure is developed for human face recognition, namely, weighted matrix distance. The key difference between this metric and the standard distances is the use of matrices and weights rather than the vectors only. The two feature matrices are obtained by two-dimensional principal component analysis (2DPCA). The weights are the inverse of the eigenvalues sorted in decreasing order of the covariance matrix of all training face matrices. Experiments are performed under illumination and facial expression variations using four face image databases: ORL, Yale, PF01 and a subset of FERET. The results demonstrate the effectiveness of the proposed weighted matrix distances in 2DPCA face recognition over the standard matrix distance metrics: Yang, Frobenius and assembled matrix distance (AMD).
\end{abstract}

Key words: classification, similarity measure, two-dimensional PCA, weighted matrix distance, human face

\section{Introduction}

Face images classification involves comparing an image with all the training face images and assign it to the closest face (class) in term of similarity measure. Several classifiers were used in the face recognition domain such as neural networks, Hidden Markov Models (HMM) and Support Vector Machines. One of the simplest and most popular, from the first application (Turk et al. 1991) to the most recent (Yang et al., 2004) (Rouabhia et al., 2008) is the Nearest Neighbor (NN) classifier with the Euclidean metric. However, the NN classifier depends crucially on the metric used to determine distances between the feature vectors that influence the recognition system performances. In the classical face recognition systems, images of faces are represented as high-dimensional pixel arrays that are time and memory consuming. To overcome this problem, Yang (Yang et al., 2004) has proposed two-dimensional principal component analysis (2DPCA) to provide feature extraction and dimensionality reduction and the matrix distance called the Yang distance. The key difference between the standard

The Authors are with Laboratoire d'Automatique et Informatique de Guelma -LAIG- Université, 8 mai 1945 de Guelma, Algérie, BP.401, Guelma, 24000, Algérie. E-mails: c_rouabhia@yahoo.fr, tebbikh@yahoo.com

Received 10.04.2011. 
distances and the Yang distance is that the first uses two feature vectors while the second uses two feature matrices. In (Zuo et al., 2005), assembled matrix distance (AMD) was proposed and tested for face and palmprint recognition. The authors have demonstrated that AMD outperforms the Yang and Frobenius distance. Inspired by the matrix distances, this paper proposes weighted distance metrics for face classification and recognition. The inverse of the eigenvalues of the total scatter matrix are used as the weights. Our objective is to evaluate and compare the efficiency of the proposed distance metric under varying illumination and facial expression. The paper is organized in six sections. The introduction followed by the second section explains the 2DPCA. Section 3 reviewed classification and some distance metrics commonly used in the face recognition domain. We introduce also the principle of weighted matrix distance metrics. Section 4 details all the image databases used for experimental tests. Simulations results and comments are presented in the fifth section. Finally, we conclude the paper.

\section{Overview of two-dimensional principal component analysis (2DPCA)}

Principal component analysis (PCA) is well-known linear feature extraction and dimensionality reduction method widely used in many applications involving highdimensional data such as face recognition. Recently, two-dimensional principal component analysis (2DPCA) was developed for face representation (Yang et al., 2004). It is straightforward image projection technique based on 2D image matrix rather than 1D image vectors. 2DPCA is essentially PCA performed on the rows of all the face images that maximizes the total scatter matrix. The key difference between 2DPCA and PCA is that each principal component of 2DPCA is a vector whereas the principal component of PCA is a scalar. 2DPCA outperforms PCA because:

i) it reduces a time to determine covariance matrix and space complexities,

ii) it keeps $2 \mathrm{D}$ spatial information,

iii) it uses a nearest neighbor classifier between two feature matrices (the Yang distance) rather than two vectors.

2DPCA arises in two steps; in the training stage, all training face images are projected to obtain feature matrices. Whereas in the test stage, each new face image is projected via the matrix projection determined in the training stage. The following steps summarize the 2DPCA algorithm:

1. Compute the total mean: $\bar{X}=\frac{1}{\mathrm{M}} \sum_{j=1}^{\mathrm{M}} X_{j}$ where $M$ is the total number of images in the training set.

2. Compute the image covariance matrix: $G_{t}=\frac{1}{\mathrm{M}} \sum_{j=1}^{\mathrm{M}}\left(X_{j}-\bar{X}\right)^{T}\left(X_{j}-\bar{X}\right)$. 
3. Form a matrix $R=\left[R_{1} R_{2} \cdots R_{d}\right]$. Columns are the eigenvectors of $G_{t}$ in decreasing order of eigenvalues.

4. Feature extraction: $Y_{j}=X_{j} R$ for $j=1, \cdots, \mathrm{M}$.

The obtained $(n \times d)$ matrix $Y_{j}$ which will be used for classification is called the feature matrix or feature image of the image sample $X_{j}$.

\section{Similarity measures}

\subsection{Some usual vectors-based distances}

In this section, we present the most usual distance measures in the classification filed. Denote $X=\left[x_{1}, x_{2}, \ldots, x_{n}\right]$ and $Y=\left[y_{1}, y_{2}, \ldots, y_{n}\right]$ two vectors in $\Re^{n}$. Minkowski distances between $X$ and $Y$ are defined as:

$$
d_{p}(X, Y)=\sqrt[p]{\sum_{i=1}^{n}\left|x_{i}-y_{i}\right|^{p}}, \quad p \in N .
$$

The Manhattan distance is obtained with $p=1$ :

$$
d_{1}(X, Y)=\sum_{i=1}^{n}\left|x_{i}-y_{i}\right|
$$

and the Euclidean distance is with $p=2$ :

$$
d_{2}(X, Y)=\sqrt{\sum_{i=1}^{n}\left(x_{i}-y_{i}\right)^{2}} .
$$

In the Eigenfaces (Turk et al., 1991), the most popular face recognition algorithm, is based on the Euclidean distance and the eigenvalues of the covariance matrix as follows:

$$
d_{2}(X, Y)=\sqrt{\frac{1}{\lambda_{i}} \sum_{i=1}^{n}\left(x_{i}-y_{i}\right)^{2}} .
$$

\subsection{Matrices-based distances}

Given two feature matrices obtained using 2DPCA $Y_{i}=\left[y_{1}^{(i)} y_{2}^{(i)} \cdots y_{d}^{(i)}\right]$ and $Y_{j}=$ $\left[y_{1}^{(j)} y_{2}^{(j)} \cdots y_{d}^{(j)}\right]$. The distance is measured between two feature matrices $Y_{i}$ and $Y_{j}$ so if $d\left(Y, Y_{l}\right)=\min _{j} d\left(Y, Y_{j}\right)$ and $Y_{l} \in C_{l}$ then $Y$ is assigned to the class $C_{l}$. 


\section{a. Yang distance}

In 2004, Yang (Yang et al. 2004) has proposed the Yang distance for face images classification. Experimental tests showed the high performances of matrix distancebased 2DPCA over the classical Euclidean distance-based PCA (eigenfaces). The Yang distance is defined as follows:

$$
d\left(Y_{i}, Y_{j}\right)=\sum_{k=1}^{d}\left(\sum_{h=1}^{n}\left(y_{h k}^{(i)}-y_{h k}^{(j)}\right)^{2}\right)^{1 / 2} .
$$

\section{b. Frobenius distance}

The Frobenius distance is a metric derived from the Frobenius matrix norm as demonstrated in (Zuo et al. 2005). It was used in (Yang et al., 2002) and defined as:

$$
d\left(Y_{i}, Y_{j}\right)=\sum_{k=1}^{d} \sum_{h=1}^{n}\left(y_{h k}^{(i)}-y_{h k}^{(j)}\right)^{2} .
$$

\section{c. AMD distancee}

Zuo (Zuo et al. 2005) has proposed the 'Assembled Matrix Distance metric: AMD' and demonstrated that both the Yang and the Frobenius distances are a special cases of AMD for $p=1$ and $p=2$ respectively. In (Zuo et al. 2005) parameter $p$ is set equal to 0.125 . AMD is defined as follows:

$$
d\left(Y_{i}, Y_{j}\right)=\left(\sum_{k=1}^{d}\left(\sum_{h=1}^{n}\left(y_{h k}^{(i)}-y_{h k}^{(j)}\right)^{2}\right)^{p / 2}\right)^{1 / p} \quad p \succ 0 .
$$

\subsection{The weighted matrix distance metrics}

We propose in this paragraph a novel weighted matrix distance metric based on feature matrices obtained by 2DPCA. Each column of the feature matrix is multiplied by the inverse of the eigenvalue corresponding to the eigenvector of the covariance matrix in decreasing order. The weighted matrix distance is defined as follows:

$$
d\left(Y_{i}, Y_{j}\right)=\left(\sum_{k=1}^{d_{1}}\left(\frac{1}{\lambda_{k}} \sum_{h=1}^{n}\left(y_{h k}^{(i)}-y_{h k}^{(j)}\right)^{2}\right)^{p / 2}\right)^{1 / p}
$$

We call, hereafter, weighted Yang distance for $p=1$ and weighted Frobenius distance for $p=2$. For $p \in] 0,1$, the distances are called the weighted fractional matrix distance metrics. For weighted AMD metric, we set $p=0.125$. 


\section{Experiments and analysis}

In this section, we experimentally test and evaluate the performance of the proposed weighted matrix distance metrics using four well-known face databases: ORL, YALE, PF01 and a subset of FERET that are publicly available. Firstly, 2DPCA is applied on the face mages to feature extraction and dimension reduction and then classification step is performed using a matrix distance metrics with the Nearest Neighbor (NN) classifier. We have investigated the impact of the parameter $p$ (eqn. 8) and the impact of the number of principal component vectors (eigenvectors) $d$ on classification accuracy. For all plots presented in the next sections, the x-axis denotes the values of $d$ and the y-axis denotes the recognition rate accuracy.

\subsection{Preprocessing}

The preprocessing techniques applied for a subset of FERET database are resizing, rotation correction, cropping and histogram equalization. The eyes coordinates are manually located and images rotated to make the line between the two eyes parallel to the horizontal axis.
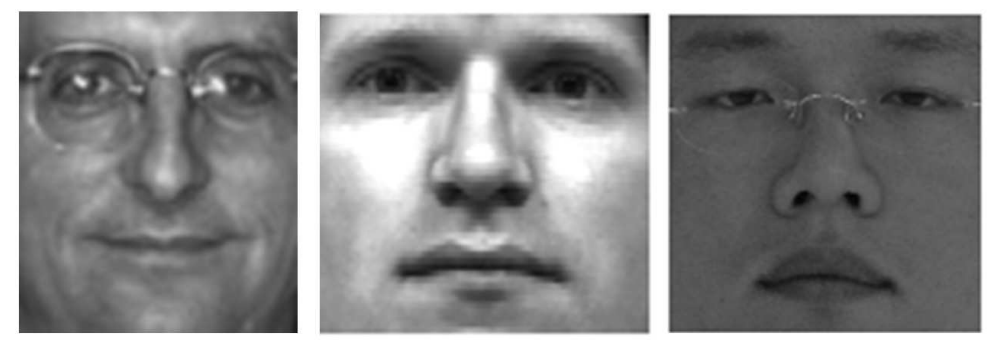

Figure 1. Examples of cropped images.

Then images are resized using the bilinear method to fix the inter-ocular distance to 43 pixels. The final cropped face images are scaled to $(90 \times 79)$ pixels. Histogram equalization is performed on the cropped FERET and PF01 $(119 \times 115$ pixels $)$ images before they are presented to subspace technique. Whereas the YALE face images are cropped to $(101 \times 106)$ pixels and used without histogram equalization. The original ORL images are used without pre-processing.

\subsection{Experiments on the ORL database}

The web address http://www.uk.research.att.com/facedatabase.html directs to ORL (Olivetti Research Laboratory). It is used to evaluate facial recognition systems under conditions where there are variations in illumination conditions, facial expressions (smiling/no smiling, open/closed eyes), scale and occlusion (glasses/no glasses). The 40 ORL's persons are shown in Fig. 2; each one has 10 different images. All images are 
greyscale and normalized to a resolution of $(112 \times 92)$ pixels. ORL was partitioned into two sets of 200 images each one; the first is used for training and the other for testing.

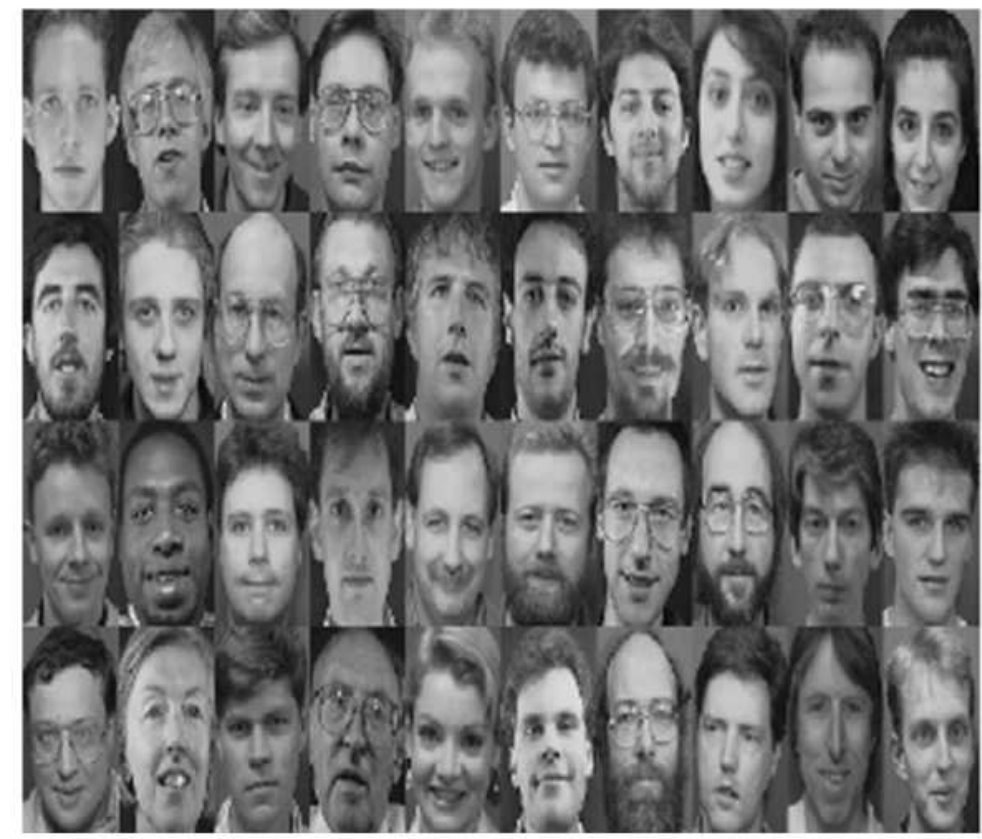

Figure 2. Face images of 40 persons from the ORL database.

We investigate, in this section, the effect of the parameter $p$ of the AMD metric (eqn. 8 ) and compare the recognition accuracy of different distances metric. We have, firstly, applied 2DPCA on the original images to feature extraction and dimension reduction and then performed the classification step. For all experiments, we have varied the parameter $p: p=0.125, p=0.3, p=0.5, p=1$ and $p=2$.

Figure 3 shows that the Yang and Frobenius distances are less dependent of the eigenvectors number compared with the others one. However the fractional distances give higher recognition rate with less number of eigenvectors. We conclude as (Zuo et al., 2005) that the recognition rate decreases as parameter $p$ increases.

Figure 4 shows the evolution of the recognition rates obtained with weighted matrix distance metric. It is very clear that the weighted Frobenius distance is also the less efficient but the weighted distance for $p=0.125$ is the best one.

Table 1 compares and summarizes the best recognition rate achieved for different values of $p$. The number of the eigenvectors used corresponding to the best recognition rate is given between parentheses.

Table 2 compares our result to those of (Bengherabi et al. 2008) and (Rouabhia et al. 2011) using ORL database. In (Bengherabi et al. 2008) a comparative study of three methods 2DPCA, DiaPCA and Dia2DPCA in DCT (Discrete Cosine Transform) 


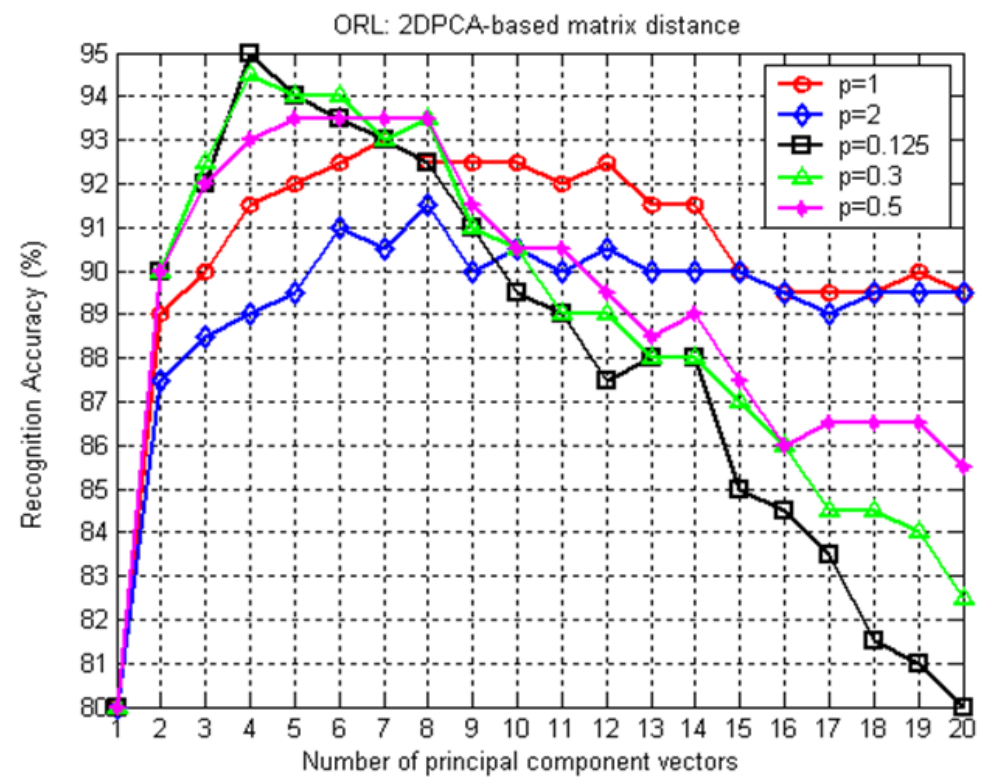

Figure 3. Recognition accuracy of different 2DPCA-based matrix distance metrics on the ORL database.

Table 2. Comparison of the top recognition accuracy (\%) of AMD vs weighted AMD.

\begin{tabular}{|c|c|c|c|c|c|}
\hline & $p=0.125$ & $p=0.3$ & $p=0.5$ & $p=1$ & $p=2$ \\
\hline AMD & 95.00 & 94.50 & 93.50 & 93.00 & 91.50 \\
& $(112 \times 4)$ & $(112 \times 4)$ & $(112 \times 5)$ & $(112 \times 7)$ & $(112 \times 8)$ \\
\hline Weighted & 95.50 & 94.50 & 94.50 & 93.00 & 90.00 \\
AMD & $(112 \times 4)$ & $(112 \times 4)$ & $(112 \times 4)$ & $(112 \times 4)$ & $(112 \times 4)$ \\
\hline
\end{tabular}

domain was conducted for face recognition. The two-dimensional DCT transform has been used as a feature extraction step, then 2DPCA, DiaPCA and DiaPCA+2DPCA are applied to $w \times w$ upper left block of the global 2D DCT transform matrix of the original images. In (Rouabhia et al. 2011), fusion of two feature matrices obtained using twodimensional PCA and two-dimensional LDA (2DLDA) was proposed and compared with the classical 2DPCA and 2DLDA. It was demonstrated experimentally that the fusion outperforms 2DPCA and 2DLDA using the AMD metric.

We have obtained $95.50 \%$ as (Bengherabi et al. 2008) and (Rouabhia et al. 2011) but with less effort and time in the feature extraction step. 


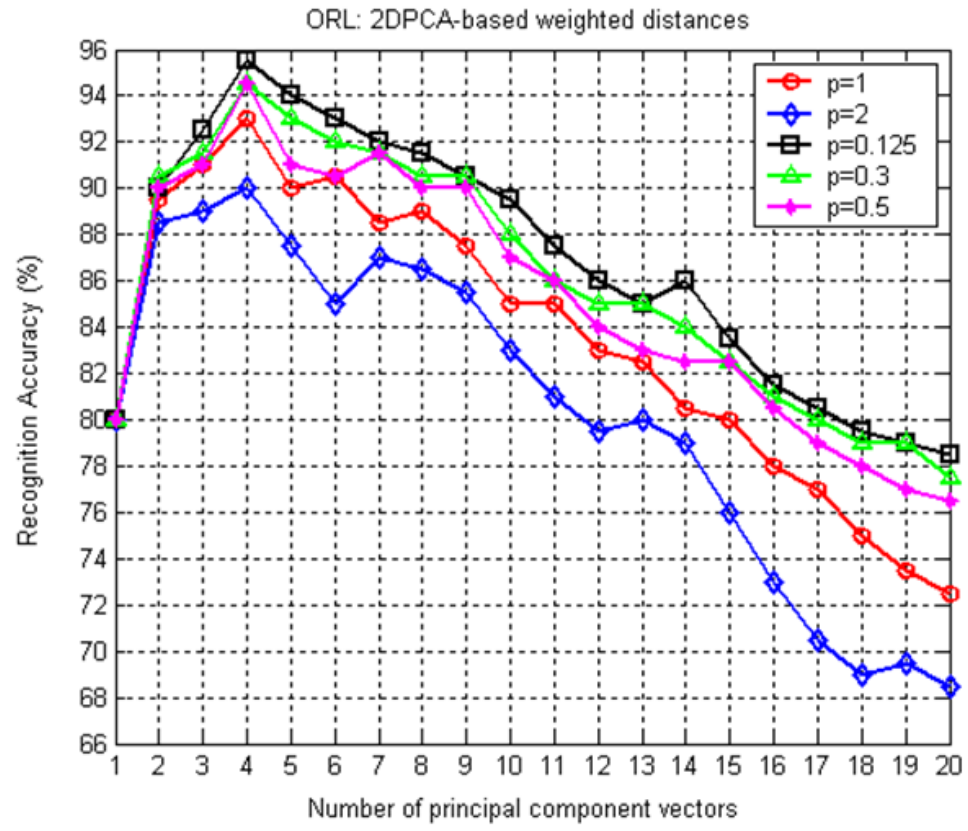

Figure 4. Recognition accuracy of different 2DPCA-based weighted matrix distance metrics on the ORL database.

Table 3. Comparison of the top recognition accuracy (\%) of different methods.

\begin{tabular}{|c|c|c|c|c|c|}
\hline 2DPCA & 2DLDA & $\begin{array}{c}\text { DiaPCA+ } \\
\text { 2DPCA }\end{array}$ & DiaPCA & Features Fusion & $\begin{array}{c}\text { Proposed } \\
\text { weighted } \\
\text { AMD }\end{array}$ \\
\hline 94.50 & 94.50 & 95.50 & 95.50 & 95.50 & 95.50 \\
\hline
\end{tabular}

\subsection{Experiments on the YALE database}

Under the web address http://cvc.yale.edu/projects/yalefaces/yalefaces.html), the Yale Face database is available. The database is constructed at the Yale center of computational vision and control, and contains 165 grayscale images of 15 subjects in a variety of conditions including with and without glasses, illumination variation and changes in facial expression (normal, happy, sad, sleepy, surprise and wink). Figure 5 shows the original YALE images used in our study. All of the left-light and right-light images were excluded because they differed significantly from what we expect.

The YALE database has been extensively used by other researchers in the field using the leave-one out strategy (Yang et al. 2004) (Belhumeur et al., 1997) (Gao et al., 2002). 


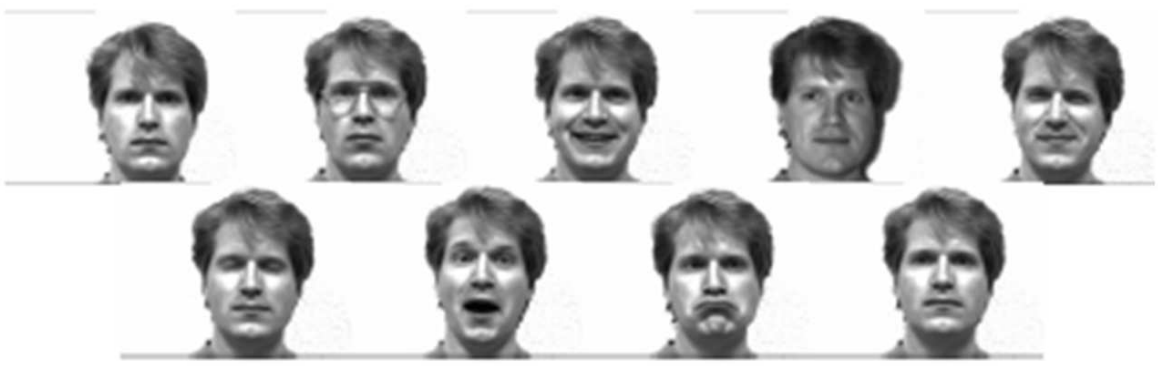

Figure 5. Example images of one person from the YALE database.

So, we have adopted the same strategy in which one image of each person is removed from the data set and all of the remaining face images are used for training. Nine (9) foldcross validations were conducted and the final recognition rate reported is the average of all.

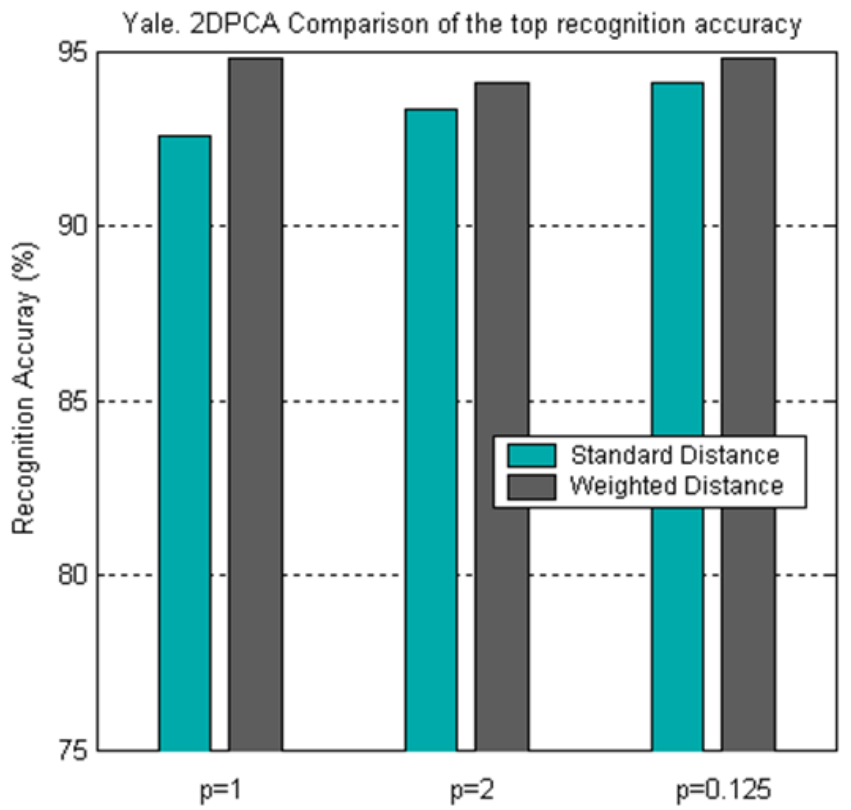

Figure 6. Comparison of the top recognition rates of different standard and weighted matrix distance metrics on the Yale database.

As shown in Fig. 6, standard distance metrics (Yang, Frobenius and AMD) were compared with the weighted matrix distances. For all the cases, the highest recognition rate is $94.81 \%$ obtained with the weighted matrix distances for $p=1$ and $p=0.125$, for the standard Yang distance is $92.59 \%$ and standard Frobenius distance is $93.33 \%$. 


\subsection{Experiments on the PF01 database}

The asian face image database PF01 (http://nova.postech.ac.kr) contains 107 of persons (56 males and 51 females) with 17 variations: facial expressions, illumination and pose variations. In (Visani et al., 2005) only a subset containing 75 persons not wearing eyeglasses under neutral illumination conditions were used. In our study, we have expected all the 107 persons under neutral illumination. Figure 7 shows the original facial expressions images (normal, smile, surprised, unpleasant and closed eye) used.
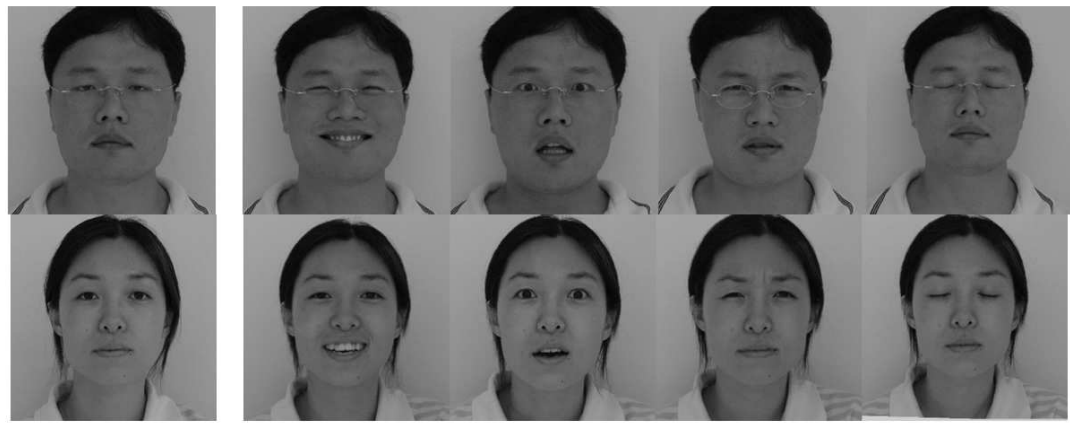

Figure 7. Examples of facial expressions from the PF01database.

Experiments tests were conducted using a neutral expression for training and two sets data for testing. The first one contains the smile expression only and the second contains all the facial expressions. Recognition accuracy evolutions of the classical AMD and weighted AMD are presented in Fig. 8 for $p=0.125$.

For smile expression, the best recognition rates obtained using classical AMD and weighted AMD are, respectively, $83.17 \%$ and $85.04 \%$ for 16 component vectors. For all facial expressions, the best recognition rates obtained using classical AMD and weighted AMD are, respectively, $75.93 \%$ and $76.40 \%$ for 16 component vectors. Classical Yang, Frobenius and AMD metrics were also compared with the weighted AMD metric. As shown in Fig. 9 the weighted AMD metric outperforms all others distance metrics.

\subsection{Experiments on the FERET database}

We have randomly selected a subset of 200 persons from FERET database (http://www.itl.nist.gov/iad/humanid/feret/), each of whom has two frontal view images: fa was used for training and fb for testing. The face images used are very diversified; there are faces with different race, gender, age, expression, illumination, occlusion, scale, etc., which greatly increases the difficulty of the recognition task.

Figures 11 and 12 compare the recognition accuracy evolution of the standard Yang and Frobenius distances with the weighted matrix distances. The highest recognition rate is $85.00 \%$ for the weighted Yang distance and $84.50 \%$ for weighted Frobenius distance. For the standard matrix distances, the highest recognition rate is $84.50 \%$ for Yang dis- 


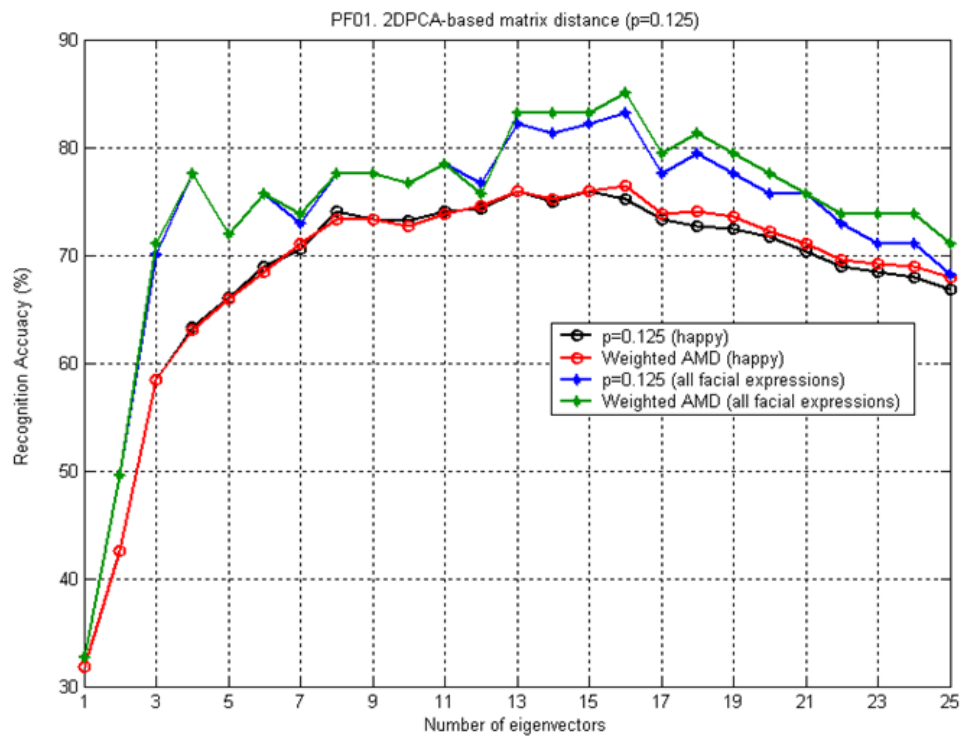

Figure 8. Recognition rate comparison between AMD and weighted AMD metrics using smile and all facial expressions.

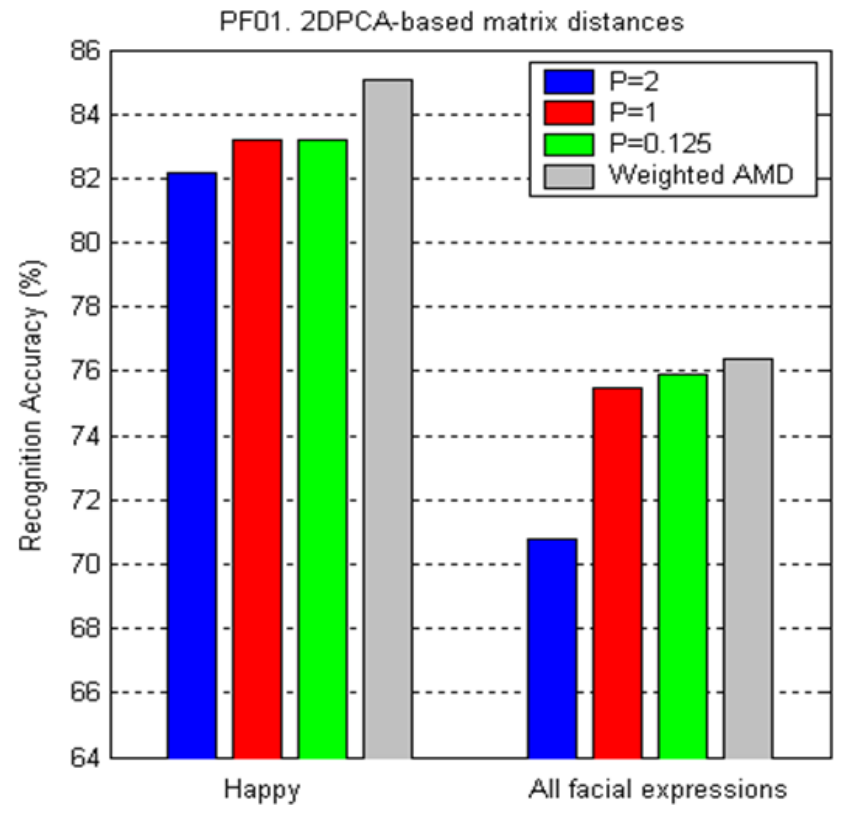

Figure 9. Comparison of the top recognition rates of different matrix distance and the weighted AMD metrics using smile and all facial expressions. 


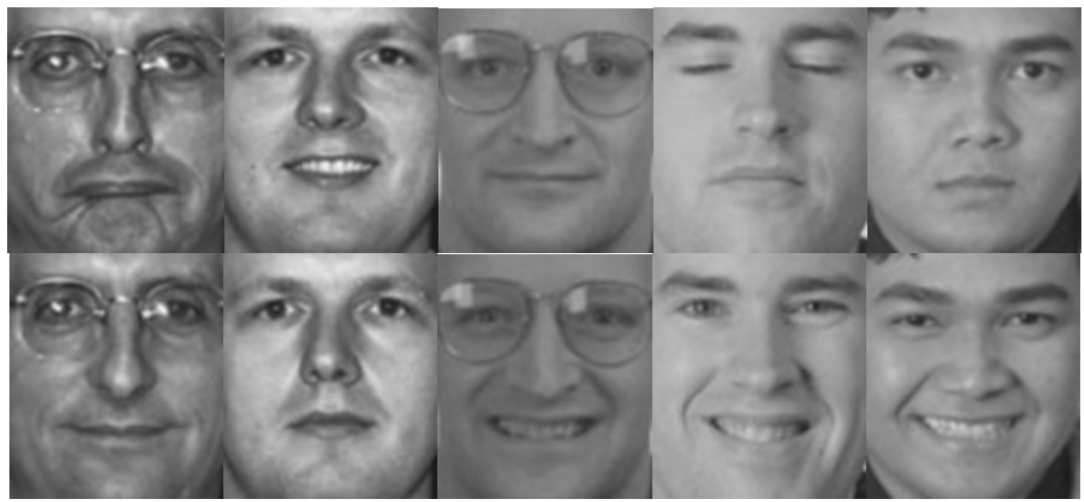

Figure 10. Examples of some cropped face images from FERET database.

tance and 82.50 for Frobenius distance. For AMD metric and weighted AMD metric, we have obtained the same recognition rate $84.00 \%$.

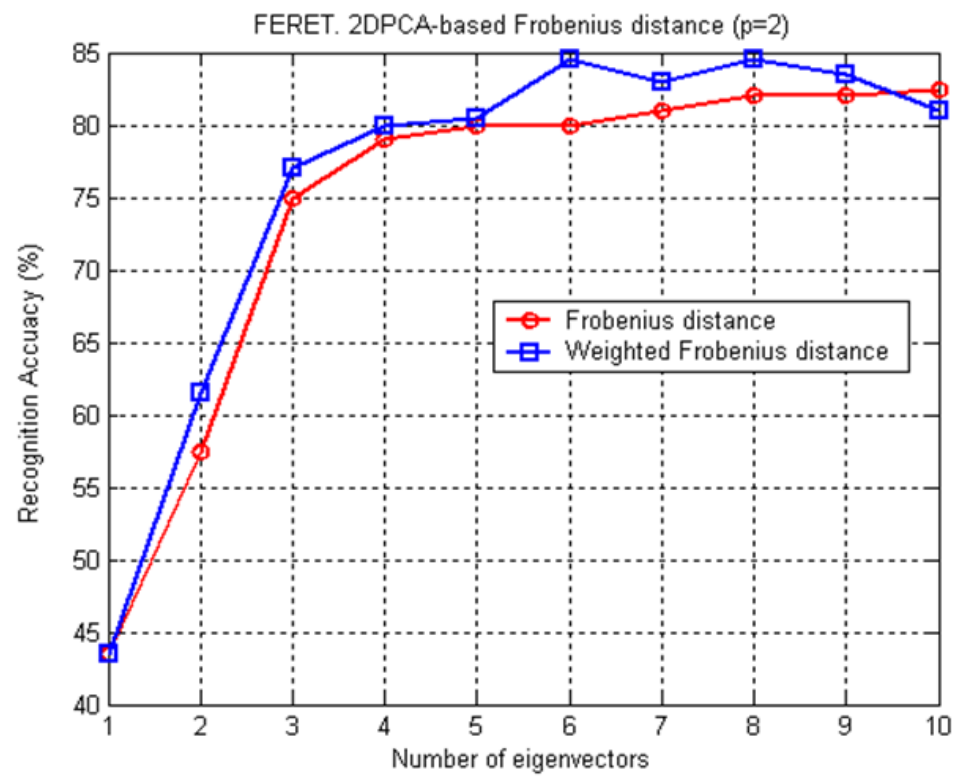

Figure 11. Recognition rate comparison between standard Frobenius and weighted Frobenius distances.

Table 3 compares our results with the state of the art using a subset of FERET database. In (Zhang et al.,2005), two-directional two-dimensional PCA ((2D)²PCA)was compared with 2DPCA and alternative 2DPCA using a partial FERET face database 


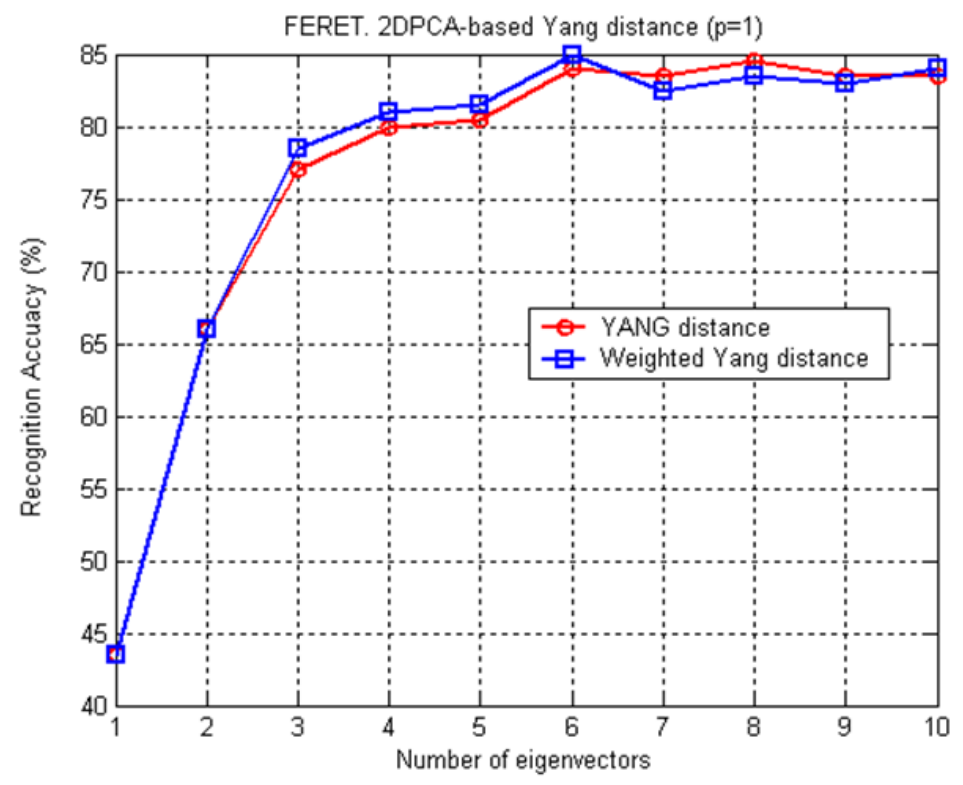

Figure 12. Recognition rate comparison between standard yang and weighted Yang distances.

Table 4. Comparison of top recognition accuracy (\%) obtained by different methods.

\begin{tabular}{|c|c|c|c|c|}
\hline \multirow{2}{*}{ 2DPCA } & \multirow{2}{*}{$\begin{array}{c}\text { Alternative } \\
\text { 2DPCA }\end{array}$} & \multirow{2}{*}{ (2D) $)^{2}$ DPCA } & \multicolumn{2}{|c|}{$\begin{array}{c}\text { Proposed weighted matrix } \\
\text { distances }\end{array}$} \\
\cline { 4 - 5 } & & $\begin{array}{c}\text { weighted } \\
\text { Frobenius }\end{array}$ & $\begin{array}{c}\text { weighted } \\
\text { Yang }\end{array}$ \\
\hline 84.5 & 84.5 & 85.0 & 84.50 & 85.0 \\
$(13 \times 60)$ & $(14 \times 60)$ & $(13 \times 14)$ & $(6 \times 79)$ & $(6 \times 79)$ \\
\hline
\end{tabular}

that comprises 400 gray-level frontal view face images from 200 persons (71 females and 129 males), each of which is cropped with the size of $60 \times 60$.

\section{Conclusion}

We have proposed a new similarity measure for classification and face recognition: weighted matrix distance metric. This distance is calculated between two 2DPCA feature matrices. The weights are the inverse of eigenvalues of the covariance matrix of all 
training face matrices. To test and evaluate the performance of the proposed distance, a series of experiments were carried out under illumination and facial expression variations using ORL, Yale, PF01 and FERET databases. Also, comparison of the proposed matrix distances with three popular distances is performed. The results show that the weighted matrix distances are effective for 2DPCA face recognition.

\section{References}

[1] P.N. Belhumeur, J.P. Hespanha and D.J. Kriegman: Eigenfaces vs Fisherfaces: Recognition using class specific linear projection. IEEE Trans. on Pattern Analysis and Machine Intelligence, 19(7), (1997), 711-720.

[2] $\mathrm{Ch}$. Rouabhia and H. Tebisikn: Hybrid feature extraction-based approach for partial parts representation and recognition. American Institute of Physics Proceedings (AIP), Intelligent Systems and Automation, 1019 (2008), 20-24.

[3] M.A. TuRK and A.P. PENTLAND: Eigenfaces for recognition. J. of Cognitive Neuroscience, 3(1), (1991), 71-86.

[4] J. YAng, D. Zhang, A.F. Frangi and J-Y. YANG: Two dimensional PCA: A new approach to appearance-based face representation and recognition. IEEE Trans. on Pattern Analysis and Machine Intelligence, 26(1), (2004).

[5] J. YANG and J-Y. YANG: From image vector to matrix: A straightforward image projection technique-IMPCA vs. PCA. Pattern Recognition, 35(9), (2002), 19971999.

[6] Y. GAO and M.K.H. LEUNG: Face recognition using line edge eap. IEEE Trans. on Pattern Analysis and Machine Intelligence, 24(6), (2002).

[7] W. ZUO, K. WANG and D. ZhANG: Assembled matrix distance metric for 2DPCA-based face and palmprint recognition. Proc. of 4th Int. Conf. on Machine Learning and Cybernetics, (2005), Guangzhou.

[8] M. Visani, C. Garcia and J-M. Jolion: Face recognition using modular bilinear discriminant analysis. Proc. of the Int. Conf. on Visual Information Systems, (2005), Amsterdam.

[9] Z. Daoqiang and Z. Zhi-Hua: (2D) ${ }^{2}$ PCA: Two-directional two-dimensional PCA for efficient face representation and recognition. Neurocomputing, 69 (2005), 224-231.

[10] M. Bengherabi, L. Mezai, F. Harizi, M. Cheriet and A. Guessoum: Face recognition based on 2DPCA, DIAPCA and DIA2DPCA in DCT domain. The 5th 